\title{
Left of what? The role of egocentric coordinates in neglect
}

\author{
Nicoletta Beschin, Roberto Cubelli, Sergio Della Sala, Lucia Spinazzola
}

\begin{abstract}
Objectives-Egocentric coordinate systems centred on the trunk, head, and gaze have been investigated in a patient who displays severe extrapersonal neglect and in five control subjects.

Methods-The subjects were tested with a blind tactile exploration task in five different experimental conditions in which the role of the three distinct frames of reference was individually controlled.

Results-Only the trunk centred coordinates significantly influenced the performance of the patient, therefore proving of paramount importance in determining the boundaries of the neglected field. Similar results emerged from a single word reading task, in which the patient's performance improved when the stimuli were presented to the right of his body's midline.

Conclusion-These findings point to the importance of the body centred coordinate system in determining the area of extrapersonal spatial neglect.
\end{abstract}

(F Neurol Neurosurg Psychiatry 1997;63:483-489)

Keywords: neglect; egocentric frames; trunk coordinates

USL 2, Unità

Operativa di Gallarate, Ospedale Bellini,

Somma Lombardo

(Va), Italy

N Beschin

Dipartimento di Psicologia dello

Sviluppo e della

Socializzazione,

Universita' di Padova,

Italy

R Cubelli

Neuropsychology

Research Division,

Department of

Psychology, University

of Aberdeen, UK

S Della Sala

L Spinazzola

Correspondence to: Professor S Della Sala, Department of Psychology, University of Aberdeen,

Kings College, Aberdeen $\mathrm{AB} 24$ 2UB, UK.

Received 30 October 1996 and in final revised form 7 April 1997

Accepted8 April 1997
Extrapersonal neglect refers to the deficit in attending to stimuli in one hemispace across different sensory modalities (visual, auditory, and tactile). Although it can involve different spatial dimensions (horizontal, radial, and vertical), extrapersonal neglect more often affects the left hemispace after a right cerebral lesion. ${ }^{1}$

The boundaries of the neglected space are not constant in as much as the neglect patients' performance is influenced by the relevant system of spatial coordinates: egocentric (viewer centred) or allocentric (environment centred). Double dissociating patterns of deficits between egocentric and allocentric extrapersonal neglect have been reported. ${ }^{2}$ Some confirmatory evidence of the distinction between egocentric and allocentric space also comes from studies on normal subjects. ${ }^{3}$

The egocentric (viewer centred) frame of reference defines the location of each stimulus relative to the spatial position of the subject. It is centred either on the vertical midline of the visual field, of the head, or of the trunk. ${ }^{45}$ The allocentric system is independent of the position of the observer and encompasses the environmental, the stimulus, and the object coordinates. Environment centred representa- tions specify the location of objects relative to one another. ${ }^{6-9}$ Stimulus centred coordinates code the right and left halves of each stimulus independently of its spatial location..$^{10}{ }^{11}$ Object centred frames refer to the canonic representation of the object itself independent of its actual orientation. ${ }^{12-15}$

There is good agreement in the literature that damage to any one of these three allocentric coordinate systems can yield extrapersonal neglect phenomenology. Indeed, specific neglect patterns have been seen according to the damage of the environmental, ${ }^{6}$ the stimulus centred, ${ }^{10}$ and the object centred ${ }^{11}$ frame of reference. On the other hand, within the frame of egocentric neglect, the specific contribution of each coordinate system in determining the boundaries of the neglected hemispace is still a matter of debate. ${ }^{16-18}$ Whether all or some of the egocentric coordinate systems (gaze, head, and trunk) are involved in separating the "good" ipsilesional from the "neglected" contralesional hemispace is open to question.

Karnath et $a l^{18}$ analysed the effect of head and trunk position in four patients with neglect. They found that the patients' saccadic reaction times for stimuli presented in the left visual field were significantly reduced when their trunk was rotated by $15^{\circ}$ toward the left. Therefore, these authors maintained that the border between the attended and the neglected hemispace is determined by the coordinate system centred on the trunk: the system centred on the head not being relevant. Karnath et al were able to replicate his findings in a subsequent study. ${ }^{19}$ The role of the trunk coordinate system is supported by Chokron and Imbert ${ }^{17}$ who tested a neglect patient with a pointing task. Their blindfolded patient was requested to point straight ahead in three different experimental conditions: head and trunk aligned at $0^{\circ}$, and trunk rotated by $15^{\circ}$ to the right or to the left of the patient's head. In this latter condition the patient's neglect disappeared.

The performance of the patient reported by Kooistra and Heilman ${ }^{20}$ also underlined the importance of the trunk coordinates in eliciting neglect phenomena. In fact, her apparent left visual field defect abated when her gaze was directed $30^{\circ}$ to the right, such that stimuli in her left visual field fell to the right of the body's midline.

A contrasting view emerged from the group study of Bisiach et al. ${ }^{16}$ They found that both trunk and head coordinate systems were relevant in determining the performance of 
neglect patients tested with a blind tactile exploration task.

None of these studies tested the relative role of the gaze. Therefore the specific role of the coordinate system centred on the gaze remains to be specified. Moreover, the lack of studies investigating the various egocentric frames within the same experimental paradigm calls for further analysis.

The aim of the present study is to systematically investigate the role of the three egocentric coordinate systems in determining the borders of the neglected hemispace. To this end we tested a patient showing severe visuospatial extrapersonal neglect on a blind tactile exploration task, as well as five control subjects.

\section{Methods}

SUBJECTS

A patient presenting with severe neglect and five age matched healthy controls gave formal consent to enter the study. The healthy volunteers were selected from the same sociocultural background of the patient. No other patients or controls have been approached and refused testing.

A 65 year old retired tailor with five years of formal education had a CT documented ischaemic stroke in the territory of the right middle cerebral artery. At the time of testing, three months after onset, he still showed left hemiplegia, hemianaesthesia, and hemianopia. $\mathrm{He}$ was fully aware of his motor and sensory impairments.

The patient performed above cut-off values on standardised tests of global cognitive level and verbal intelligence: the Italian version ${ }^{21}$ of the mini mental state examination ${ }^{22}$ and the verbal judgements test. ${ }^{23}$ Age and education adjusted scores were respectively $25.3 / 30$ (cutoff score $=23.8$ ) and 51.5/60 (cut-off=33).

In addition, the patient was given a clinical battery to detect the presence of extrapersonal neglect. On cancellation tasks he crossed out only the extreme right stimuli: $12 / 40$ lines, ${ }^{24}$ $10 / 104$ letters, ${ }^{25} 5 / 35$ bells. ${ }^{26}$ On copying geometric shapes ${ }^{27}$ and object pictures ${ }^{28}$ he reproduced only the right sided stimuli in a multiple array or the right half (sometimes less) of single item pictures. The patient showed left neglect also in drawing from memory tasks. ${ }^{28}$ On coloured progressive matrices ${ }^{29}$ he chose 21 alternatives from the right and only two from the left. The right-left difference (19) was far from the cut-off score of $8,{ }^{30}$ thus showing an abnormal right position preference. On the Wundt-Jastrow test ${ }^{31}$ he produced six unexpected responses out of 20 stimuli on the left and only one on the right (cut-off: right-left difference score $=2$ ). When asked to bisect 10 $20 \mathrm{~cm}$ long lines, the patient displaced the central point consistently toward the right extremity (mean displacement error $7.1 \mathrm{~cm}$ (SD 1.8)).

In conclusion, the patient showed a severe left neglect in a wide variety of visuospatial tasks. He was anosognosic for his neglect, even when formally tested by means of an ad hoc questionnaire (I H Robertson, with permission, ESCAPE project). This questionnaire comprises 20 items, 10 questions to be

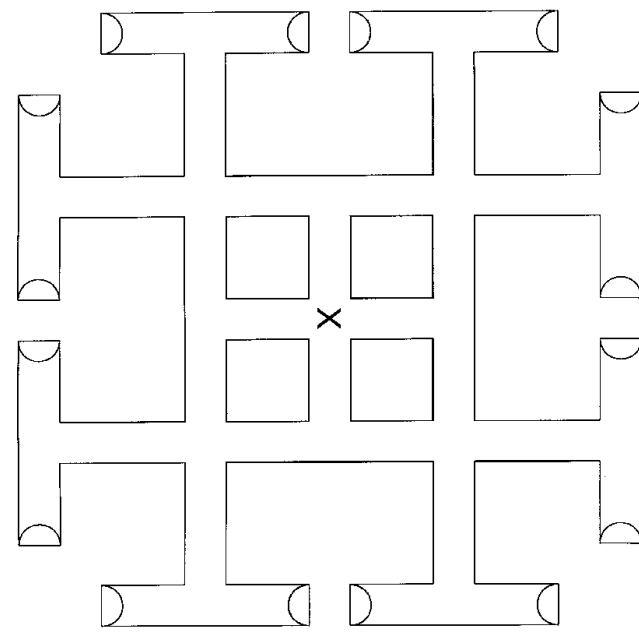

Figure 1 Schematic representation of the device used for the tactile exploration task.

answered by the patient (for example, "Do you feel you have difficulties washing the left side of your body or face?") and 10 to be filled in by the examiner (for example, "Do you think that the patient has difficulties washing the left side of his or her body or face").

\section{TACTILE EXPLORATION TASK}

Device

The apparatus was the same as used by Beschin et al. ${ }^{32}$ It consisted of a $33 \mathrm{~cm}$ long square maze cut into a wooden rectangular board $40 \mathrm{~cm}$ wide and $50 \mathrm{~cm}$ long. The maze was a modified version of that utilised by De Renzi et al, ${ }^{33}$ with $3 \mathrm{~mm}$ deep and $2 \mathrm{~cm}$ wide symmetric alleys. Four alleys spread from the centre of the maze branching into eight lateral arms, each of which ends in a $10.5 \mathrm{~cm}$ segment at the extremes of which a hollow can easily host the marble used as a target. The distance to cover from the centre to reach each of the 16 target positions was exactly the same-that is, $30 \mathrm{~cm}$ (fig 1).

\section{Testing procedure}

The patient and the control subjects each sat on a special adjustable chair in front of a table on to which the testing board was pasted. The subjects' head was supported by a rotating head rest and the chin was fixed to allow controlled alignment of the egocentric coordinates. The distance of the testing device from the subjects' body was individually adjusted to minimise leaning back and forward to reach the extremities of the maze. The maze was covered throughout the experiment to prevent visual control. The task consisted in finding, as fast as possible, using the right hand, a marble (diameter $2.4 \mathrm{~cm}$ ) placed at the extremities of one of the hollows, always starting from the centre of the maze. The time limit for each trial was 120 s. The timer was started when the subject moved his forefinger from the centre of the maze and it was stopped when the marble was touched. Any failure to locate the target within the time limit was scored as an omission, and the maximum time of $120 \mathrm{~s}$ was computed for that trial. Throughout the experiment, subjects maintained a fixation point ( $5 \mathrm{~mm}$ spotlight). 


\section{Experimental condition}

1
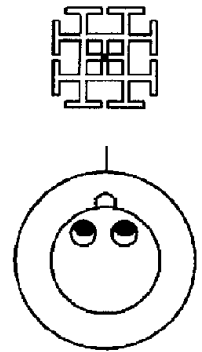

2

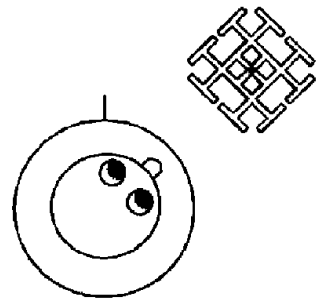

3

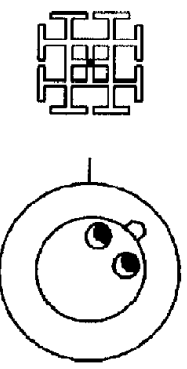

4
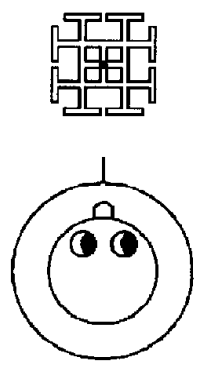

5

Frame of reference
Trunk Head Gaze

L-R

R

L-R

L-R

L-R

L-R

$\mathrm{R}$

L
Gaze
Strictly speaking, the task is a blind manual search; however we labelled it "tactile exploration" as this is the standard term used in the literature. $^{32}$

The whole test comprised five different conditions (fig 2).

\section{Condition 1 (baseline condition)}

The apparatus was placed in front of the subject. The vertical midlines of the visual field, head, and trunk were aligned.

\section{Condition 2}

The maze was displaced $30^{\circ}$ to the right of the trunk. The head and the gaze were aligned with the centre of the apparatus.

\section{Condition 3}

The apparatus was placed in front of the subject aligned to the trunk's midline; the head and the gaze were kept $30^{\circ}$ on the right.

\section{Condition 4}

The apparatus was placed in front of the subject. The vertical midlines of the head and trunk were aligned with the centre of the maze. The gaze was maintained on the right.

\section{Condition 5}

The maze was displaced $30^{\circ}$ to the right of the trunk and the head. The gaze was aligned to the centre of the apparatus.

Each experimental condition consisted of 16 randomised trials (one for each of the 16 possible positions) giving rise to a score range of $0-8$ for each side (left and right). Presentation of the stimuli were balanced- in blocks of fouracross the different conditions to avoid any fatigue effect. Both accuracy and searching time were measured. To increase reliability, the patient was tested three times.

In our experimental paradigm we made the assumption that it does not matter whether the effect of orientation is tested by looking for improvement of neglect (by presenting the apparatus to the right of the coordinate systems under investigation) or for its further deterioration (by placing the apparatus to the left of the coordinate systems under study). The reasons for this choice are both theoretical and practical. Firstly, a similar design was adopted in previous work, ${ }^{15}$ and this allows for comparison of findings. Moreover, from a theoretical point of view, there is no reason to assume that if the number of omissions and searching time do not decrease when the device is placed to the right of, say, the trunk, they should increase when the stimuli are placed to its left. From a practical point of view, it is very difficult for both normal subjects and brain damaged patients to explore a maze placed to the left of their body midline using their right hand and not rotating the trunk.

\section{Predictions}

The patient should show neglect in condition 1. If the trunk centred frame of reference played a part, his neglect was expected to decrease in conditions 2 and 5 . If neglect were 


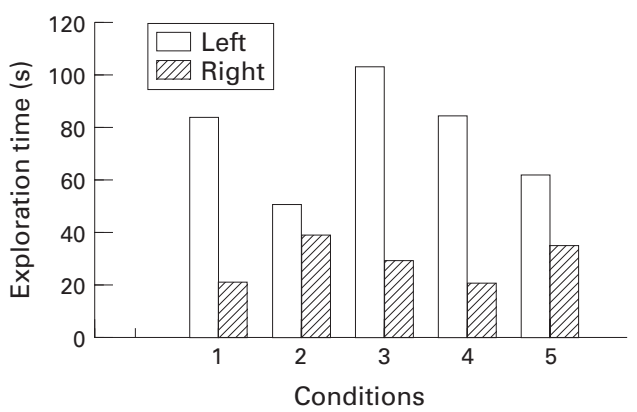

Figure 3 Mean searching times in the left and right halves of the maze in the five experimental conditions obtained by the patient.

gaze centred, his performance should worsen in conditions 3 and 4 . If the neglect borders were determined by head centred coordinates, the patient should perform better in condition 5 than in condition 2 and worse in condition 3 than in condition 4. Finally, if no difference were to be detected across conditions his neglect found in condition 1 had to be considered exclusively allocentric - that is, array centred neglect.

\section{Results}

Searching times for each trial were analysed subject by subject, by means of a series of ANOVAs. In all instances the $F$ values for side (right and left), conditions (experimental conditions 1 to 5) and their interaction fell far short of significance. The control subjects were also considered as a group, and the searching $\times$ data analysed by means of repeated measures ANOVA. There was no main effect of side $(F(1,4)=0.396, \mathrm{P}=0.56)$, conditions $(F$ $(4,16)=1.502, \mathrm{P}=0.25)$, or their interaction $(F$ $(4,16)=1.147, \mathrm{P}=0.37)$.

Only one control subject, $\mathrm{C} 1$, made any error. He omitted two targets, one on the left side in condition 1 , and one on the right in condition 3.

The neglect patient was tested three times, and his mean search times (fig 3) analysed by repeated measures ANOVA. This showed a main effect of the side $(F(1,14)=25.753$, $\mathrm{P}<0.001)$, a marginal effect of conditions $(F$ $(4,56)=2.491, \quad \mathrm{P}=0.053)$, and a significant interaction $(F(4,56)=6.909, \mathrm{P}<0.001)$. Search times were also analysed separately for the two halves of the maze. No significant difference among conditions emerged for the right side times $(F(4,28)=1.656, \mathrm{P}=0.19)$ whereas this difference was highly significant for the left side times $(F(4,28)=6.632, \mathrm{P}<0.001)$. Because the aim of the paper was focused on the interaction between conditions and neglect, the data have also been analysed in terms of the difference between left and right. Data have been entered in this analysis considering the difference between the mean performance of each specular position pairs in the maze. Figure 4 shows these data together with those of the control sample for comparison. The main effect of conditions was significant $(F(4,35)=4.800$, $\mathrm{P}<0.005)$. Fisher's protected least squares difference disclosed that the mean left-right search time difference in condition $2(11.5 \mathrm{~s})$

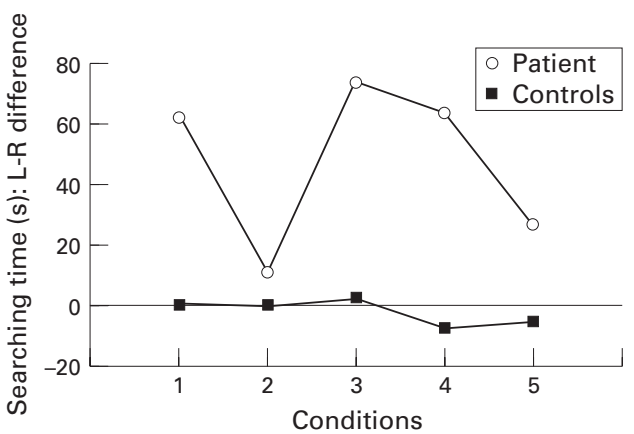

Figure 4 Mean left-right difference searching times in the five experimental conditions of the patient and the five control subjects.

Table 1 Number of omissions in the three testing sessions of the patient in the five experimental conditions according to the side of the maze $(\max =24)$

\begin{tabular}{lrl}
\hline & \multicolumn{2}{l}{ Side } \\
\cline { 2 - 3 } Conditions & Left & Right \\
\hline 1 & 12 & 0 \\
2 & 5 & 2 \\
3 & 18 & 2 \\
4 & 13 & 1 \\
5 & 8 & 3 \\
\hline
\end{tabular}

was significantly smaller $(\mathrm{P}<0.01$ in all comparisons) than that in condition $1(62.7 \mathrm{~s})$, condition $3(73.8 \mathrm{~s})$ and condition $4(63.8 \mathrm{~s})$. Condition 5 mean left-right searching times difference (27.1 s) was also significantly smaller $(P<0.05$ in all comparisons) than that of conditions 1,3 , and 4 . There was no difference between conditions 2 and 5 or between conditions 1,3 , and 4 .

Analyses of the patient's omissions (table 1) mirrored the searching time findings: side $(F$ $(1,14)=15.750, \quad \mathrm{P}<0.002), \quad$ conditions $\quad(F$ $(4,56)=3.395, \mathrm{P}<0.02)$, and their interaction $(F(4,56)=4.382, \mathrm{P}<0.005)$ were all significant. The left-right difference in the number of omissions was just short of significance ( $F$ $(4,35)=2.577, \mathrm{P}=0.054)$.

The performance of the patient on most of the clinical tests assessing extrapersonal neglect was rather poor (see case report); he managed to find targets only on the extreme right of all cancellation tasks. Therefore it has been deemed worthwhile to analyse the mean searching times and the omissions, subdividing the maze into four parts (left lateral, left medial, right medial, and right lateral) to investigate the possibility of a gradient in the improvement of performance from one condition to another. An ANOVA on searching times (raw data in table 2) showed that spatial sectors, conditions, and their interaction were all significant (respectively $F(3,12)=7.613$, $\mathrm{P}<0.005 ; F(4,48)=2.582, \mathrm{P}<0.05$, and $F$ $(4,48)=3.225, \mathrm{P}<0.002)$. Separate analyses for each condition showed a significant effect of the spatial sector only in condition 1 ( $F$ $(3,12)=15.924, \mathrm{P}<0.001), 3(F(3,12)=14.706$, $\mathrm{P}<0.001)$, and $4(F(3,12)=5.934, \mathrm{P}<0.02)$. Post hoc Fisher's least squares difference demonstrated that, in all instances, searching times for the two right sectors were significantly shorter than those for the two left sectors, 
Table 2 Mean searching times (s) of the patient in the five experimental conditions according to the four different sectors of the maze

\begin{tabular}{lclll}
\hline \multirow{5}{*}{ Conditions } & \multicolumn{4}{l}{ Spatial sectors } \\
\cline { 2 - 5 } & \multicolumn{1}{l}{$L L$} & $L M$ & $R M$ & $R L$ \\
\hline 1 & 80 & $88^{\star}$ & 17 & 25 \\
2 & 62 & 38 & 40 & 37 \\
3 & 109 & $97^{\star}$ & 27 & 32 \\
4 & 74 & $95^{\star}$ & 24 & 17 \\
5 & 70 & 54 & 22 & 47 \\
\hline
\end{tabular}

$\star \mathrm{P}<0.05$.

$\mathrm{LL}=$ left lateral; $\mathrm{LM}=$ left medial $; \mathrm{RM}=$ right medial RL = right lateral.

Table 3 Number of omissions in the three testing sessions of the patient in the five experimental conditions according to the four different sectors of the maze $(\max =12)$

\begin{tabular}{|c|c|c|c|c|}
\hline \multirow[b]{2}{*}{ Conditions } & \multicolumn{4}{|c|}{ Spatial sectors } \\
\hline & $L L$ & $L M$ & $R M$ & $R L$ \\
\hline 1 & 5 & $7^{\star}$ & 0 & 0 \\
\hline 2 & 4 & 1 & 1 & 1 \\
\hline 3 & 10 & $8^{\star}$ & 1 & 1 \\
\hline 4 & 6 & $7^{\star}$ & 1 & 0 \\
\hline 5 & 5 & 3 & 0 & 3 \\
\hline
\end{tabular}

$\star \mathrm{P}<0.05$.

$\mathrm{LL}=$ left lateral; $\mathrm{LM}=$ left medial $\mathrm{RM}=$ right medial; $\mathrm{RL}=$ right lateral.

which did not differ from one another. Also the general analysis on omissions (see table 3) yielded significant effects for spatial sectors $(F$ $(3,12)=4.669, \quad \mathrm{P}<0.05), \quad$ conditions $\quad(F$ $(4,48)=3.422, \mathrm{P}<0.02)$, and their interaction $(F(4,48)=2.176, \mathrm{P}<0.05)$. Separate ANOVAs for individual condition and post-hoc analyses mirrored the findings of searching times in showing a split half in conditions 1,3 , and 4 and no significant difference across spatial sectors in conditions 2 and 5 . However, it is clear, on inspecting tables 2 and 3 , that in conditions 2 and 5 the reduction of latencies and inaccuracies is more overt in the left medial than in the left lateral sector of the maze .

These findings taken together point to a relevant role of the trunk centred coordinate system in determining the boundaries of extrapersonal neglect (see predictions in method section).

To support this claim further evidence has been sought. The patient was asked to read aloud one after the other 37 real words printed in black on white cards. The stimuli were presented twice: on a table directly in front of the patient and at $30^{\circ}$ to the right of the trunk's midline, with the head and the gaze free to move. There was no time constraint. When the cards were centrally presented, the patient misread all 37 words, 36 errors (97\%) were classified as neglect errors according to the criteria of Ellis et $a l^{34}$ : the initial letters were omitted (for example, PORTO (harbour) $\rightarrow$ ORTO (kitchen garden)) or substituted (for example, SERVIZIO (service) $\rightarrow$ INIZIO (beginning)). The patient's performance improved considerably when the stimuli were presented in the right hemispace. In this condition he read nine words flawlessly and only 20 of the 28 paralexic errors $(71 \%)$ were considered neglect errors. The 20 words which gave rise to neglect errors in both conditions have been analysed sepa- rately. The number of letters omitted or substituted to the left of the neglect point ${ }^{33}$ has been calculated for each stimulus word: means were 5.40 and 1.95 for the central and the right position respectively. This difference was significant (paired $t$ test $(19)=8.219, \mathrm{P}<0.0001$ ).

\section{Discussion}

The findings reported in this paper suggest that the performance on a blind tactile exploration task of a patient affected by severe extrapersonal visuospatial neglect is clearly influenced by the orientation of the trunk. In experimental conditions 2 and 5 (maze aligned to either gaze or head and to the right of the trunk) the patient's performance showed no left-right difference either in latency or accuracy. On the contrary, in conditions 3 and 4 (maze centred to the trunk's midline and to the left of the gaze or head) the right-left asymmetry remains akin to that found in the baseline condition 1. This points to the egocentric nature of the patient's tactile neglect and indicates that the coordinate system centred on the trunk plays a major part in determining the boundaries of the neglected field. On the other hand, the retinotopic and head coordinates do not seem to be relevant frames of reference.

The present findings are in agreement with most of the literature ${ }^{16-18}{ }^{20}$; although this is the first instance in which the three coordinate systems have been analysed within the same experimental design. Bisiach et $a l^{16}$ attributed a role also to the gaze centred ("line of sight" in their wording) coordinates in determining the performance of neglect patients in a blind tactile exploration task. However, the difference between their data and our own is insubstantial. In the experiment reported by Bisiach et $a l,{ }^{16}$ conditions a, c, and d correspond respectively to experimental conditions 1,2 , and 3 of the present study. Inspecting the mean data they report (table 1), the similarity with our own findings is striking. In fact, in condition " $d$ " (device aligned to the trunk, head turned toward right) no overt difference emerged in the number of omissions with respect to the baseline (condition "a"). On the contrary in condition " $c$ " (apparatus to the right of the trunk, aligned to the gaze-head midline), the number of left omissions as well as the right-left difference clearly decreased. Bisiach et $a l^{16}$ considered that the remaining right-left difference in performance of their neglect patients in condition "c", when the testing device was aligned to the line of sight, could be accounted for by assuming a role of the gaze (or head). However, this line of reasoning presupposes that the right-left difference in condition "c" is still significantly indicative of neglect, an assumption that has not been confirmed by ad hoc analyses. It could well be possible to interpret the better performance in condition " $\mathrm{c}$ " as an improvement in respect to the baseline due to the position of the apparatus, $60^{\circ}$ to the right of the trunk. In any case, Bisiach et $a l^{16}$ did not deny the role of the trunk centred coordinates.

The effect of trunk centred coordinates on neglect has been reported also with different material and experimental paradigms. For 
instance, in line bisection tasks, the mean displacement error is significantly reduced when the line to be bisected is placed to the right of the body's midline. ${ }^{35-37}$

To the best of our knowledge only one paper exists in the literature in which the author denies the possibility of a body centred neglect. ${ }^{38}$ However, the data reported by Husain $^{38}$ are not watertight. He tested eight patients with neglect and presented them with a cancellation task in two experimental conditions: with the testing display in front or to the right of the patients' body midline. Husain ${ }^{34}$ did not find any difference in performance between the two conditions. It is worth underlining, however, that the patients in his study were tested very soon after the stroke, in most cases between one and 10 days, and some of them were rather old (up to 86 years). Moreover, these patients' neglect was so severe that most of them could only manage to cancel a few stimuli: in several cases less than one tenth and in some cases only one or two out of 60 . Therefore, everything considered, it is difficult to exclude an associated aspecific attentional deficit due to the poor general conditions after a very recent stroke. Furthermore, in six patients the lesion was centred on the right frontal lobe, and non-neglect-linked attentional deficits ${ }^{39} 40$ could have been a possible added cause of the patients' poor visuospatial exploration.

The results of the tactile searching task are mirrored by the findings of the reading test. When the words to be read were on the right of our patient's body midline, his neglect errors decreased considerably. In both tests, however, his performance did not recover completely, even when the stimuli were presented at $30^{\circ}$ right of the body's midline. This can be traced back to either a non-sufficient rotation of the trunk or to an allocentric component of the patient's neglect. Alternatively, the role of the gaze or head coordinates could be called into play. ${ }^{16}$ Yet this interpretation runs into difficulty in so far as the predicted deterioration in experimental conditions 3 and 4 was not found.

The manual search and the word reading are clearly different tasks and are subsumed by different cognitive functions. However, the converging performance of the patient in the two tasks is of some relevance in emphasising the role of the trunk coordinates in coding the spatial location of the stimuli to be processed.

The bulk of these results can be discussed within the frame of the model of written word and visual object recognition proposed by Hillis and Caramazza. ${ }^{11}$ This model predicts three levels of representation to interpret different patterns of neglect: retinocentric, stimulus centred, and object centred. The first level is based on egocentric coordinates. The diagnostic test consists of asking the patient to read words in different spatial locations on the page. Only in the case of egocentric neglect should the patient show a difference in performance in such a task. However, it has still to be determined whether the relevant egocentric system is literally retinocentric or whether it might be body centred, or both. Hillis and Caramazza ${ }^{11}$ mentioned a patient who showed the "retinocentric" pattern of neglect dyslexia unaffected by rotation of her trunk or head. Our patient, as reported here, points to the autonomous role of the trunk in computing stimuli before a stimulus centred or object centred representation. This seems to indicate the possibility of a double dissociation in the early level of word recognition.

A double dissociation in egocentric neglect had been postulated already in a fleeting comment by Bisiach et $a l,{ }^{16}$ who reported two patients showing neglect when the testing device was trunk centred and not when it was gaze centred and another patient showed the opposite pattern. This dissociation, and its possible anatomical correlates, ${ }^{15}$ are matters for future investigation.

In conclusion, although based on a single case $^{4142}$ and in need of replication, the findings reported in this paper allow us to claim the importance of the body centred coordinate system in determining the area of extrapersonal spatial neglect. In addition to its theoretical relevance, this notion could also have some pragmatic implications. It is possible to posit a role for postural control in the rehabilitation of neglect. For instance, teaching the patients to orient their trunk could be envisaged as a more efficient aim for a rehabilitation programme than cueing their visual scanning ability ${ }^{25}$ a speculation that calls for proper investigation.

We thank P Bates who drew the figures, R Drinkwater for editorial help, O Turnbull for his comments on an earlier version of the manuscript, and C Spivey who amended the English. We are also very grateful to the patient and his carers who consented to also very grateful to the
the extensive testing.

1 Robertson IH, Marshall JC, eds. Unilateral neglect: clinical and experimental Studies. London: Taylor and Francis, 1993.

2 Walker R. Spatial and object-based neglect. Neurocase 1995; 1:371-83.

3 Robertson LC. Covert orienting biases in scene-based reference frames: orienting priming and visual field differences. f Exp Psychol Hum Percept Perform 1995;21:707-18.

4 Jeannerod M, Biguer B. Reference egocentrique et espace representé. Rev Neurol (Paris) 1989;145:635-9.

5 Karnath HO. Disturbed coordinate transformation in the neural representation of space as the crucial mechanism leading to neglect. Neuropsychological Rehabilitation 1994;4: $147-50$

6 Calvanio R, Petrone PN, Levine DN. Left visual spatial neglect in both environment-centered and body-centered. Neurology 1987;37:1179-83.

7 Farah M J, Brunn JL, Wong AB, Wallas MA, Carpenter PA. Evidence from the neglect syndrome. Neuropsychologia 1990;22:335-47.

8 Ladavas E. Is the hemispatial deficit produced by right parietal lobe damage associated with retinal or gravitational coordinates? Brain 1987;110:167-80.

9 Mennemeier M, Chatterjee A, Heilman KM. A comparison of the influences of body and environment centred of the influences of body and environment centrect
reference frames on neglect. Brain 1994;117:1013-21.

10 Arguin M, Bub DN. Evidence for an independent stimuluscentered spatial reference frame from a case of visual hemineglect. Cortex 1993;29:349-57.

11 Hillis A E, Caramazza A. A framework for interpreting distinct patterns of hemispatial neglect. Neurocase 1995;1; 189-207.

12 Behrmann M, Moscovitch M. Object-centered neglect in patients with unilateral neglect: effects of left-right coordinates of objects. Fournal of Cognitive Neuroscience 1994;6:116.

13 Caramazza A, Hillis AE. Spatial representation of words in the brain implied by the studies of a unilateral neglect patient. Nature 1990;346:267-9.

14 Driver J, Halligan P. Can visual neglect operate in object-centred co-ordinates? An affirmative single-case study. Cognitive Neuropsychology 1991;8:475-96.

15 Young AW, Hellawell DJ, Welch J. Neglect and visual recognition. Brain 1992;115:51-71

16 Bisiach E, Capitani E, Porta E. Two basic properties of space representation in the brain: evidence from unilateral neglect. $\mathcal{F}$ Neurol Neurosurg Psychiatry 1985;48:141-4. 
17 Chokron S, Imbert M. Variations of the egocentric reference among normal subjects and a patient with unilateral among normal subjects and a patient

18 Karnath HO, Schenkel P, Fischer B. Trunk orientation as the determining factor of the "contralateral" deficit in the neglect syndrome and as the physical anchor of the internal representation of body orientation in space. Brain 1991; 114:1997-2014.

19 Karnath HO, Christ K, Hartje W. Decrease of contralateral neglect by neck muscle vibration and spatial orientation of trunk midline. Brain 1993;116:383-96.

20 Kooistra CA, Heilman KM. Hemispatial visual inattention masquerading as hemianopia. Neurology 1989;39:1125-7.

21 Measso G, Cavarzeran F, Zappala' G, et al. The mini-mental state examination: normative study of an Italian random sample. Developmental Neuropsychology 1993;9:77-85.

22 Folstein MF, Folstein SE, McHugh PR. "Mini mental state": a practical method for grading the cognitive state of patient for the clinician. F Psychiatr Res 1975;12:189-98.

23 Spinnler HR, Tognoni G, eds. Standardizzazione e taratura italiana di test neuropsicologici. Ital $\mathcal{f}$ Neurol Sci 1987; italiana

24 Albert ML. A simple test of visual neglect. Neurology 1973; 23:658-64

25 Diller L, Weinber J. Hemi-inattention in rehabilitation: the evolution of a rational remediation program. In: Weinstein EA, Friedland RP, eds. Hemi-inattention and hemisphere specialisation: advances in neurology. Vol 18. New York: Raven Press, 1977:63-82.

26 Gauthier L, Dehaut F, Joanette Y. The Bells test: a quantitative and qualitative test for visual neglect. International fournal of Clinical Neuropsyhology 1989;11:49-54.

27 Arrigon C, De Renzi E. Constructional apraxia and hemispheric locus of lesion. Cortex 1964:1:170-97.

28 Wilson BA, Cockburn J, Halligan P. Behavioral inattention test. Flempton: Thames Valley Test Company, 1988.

29 Raven JC. Guide to using the coloured progressive matrices. London: Lewis, 1965.
30 Colombo A, De Renzi E, Faglioni P. The occurrence of visual neglect in patients with unilateral cerebral disease. visual neglect in patients

31 Massironi M, Antonucci G, Pizzamiglio L, Vitale MV, Zoccolotti P. The Wundt-Jastrow illusion in the study of spatial hemi-inattention. Neuropsychologia 1988;26:161-6.

32 Beschin N, Cazzani M, Cubelli R, Della Sala S, Spinazzola L. Ignoring left and far: an investigation of tactile neglect. Neuropsychologia 1996;34:41-9.

33 De Renzi E, Faglioni P, Scotti G. Hemispheric contribution to exploration of space through the visual and tactile to exploration of space through

34 Ellis AW, Young AW, Flude BM. "Neglect dyslexia" and the early visual processing of words and non-words. Cognitive Neuropsychology 1987;4:439-64.

35 Nichelli P, Rinaldi M, Cubelli R. Selective attention and length representation in normal sucbjects and in patients with unilateral spatial neglect. Brain Cogn 1989;9:57-70.

36 Cubelli R, Pugliese M, Gabellini AS. The effect of space location on neglect depends on the nature of the task. $\mathcal{F}$ Neurol 1994;241:611-4.

37 Halligan PW, Marshall JC. Lateral and radial neglect as a function of spatial position: a case study. Neuropsychologia 1995;33:1697-702.

38 Husain $M$. Is visual neglect body-centric? f Neurol Neurosurg Psychiatry 1995;58:262-3.

39 Della Sala S, Laiacona M, Spinnler H, Ubezio C. A cancellation test: its reliability in assessing attentional deficits in Alzheimer's disease. Psychol Med 1992;22:885-901.

40 Pardo VJ, Fox PT, Raichle ME. Localization of a human system for sustained attention by positron emission tomography. Nature 1991;349:61-4.

41 Caramazza A, McCloskey $M$. The case for single-patient studies. Cognitive Neuropsychology 1988;5:517-28.

$42 \mathrm{McCloskey} \mathrm{M}$, Caramazza A. Theory and methodology in cognitive neuropsychology: a response to our critics. Cognitive Neuropsychology 1988;5:583-623. 